\title{
Exogastric leiomyomata
}

\author{
R. C. G. RusSell \\ F.R.C.S. \\ B. A. WooD \\ B.Sc., M.B., B.S.
}

Department of Surgery, The Middlesex Hospital, London, W.1

\section{Summary}

Two cases of exogastric leiomyoma are described indicating the difficulty in diagnosis of these tumours, and illustrating the success of total excision of the tumour in such cases.

THE commonest connective tissue tumours of the stomach are leiomyomata. Most of these are small intragastric tumours commonly presenting with a haematemesis or being found incidentally at necropsy. Less frequently leiomyomata are exogastric, growing to a large size and presenting as an abdominal mass.

Two cases of exogastric leiomyomata are described which illustrate the difficulties of the diagnosis and management of this condition. These tumours rarely enter the differential diagnosis of an abdominal mass, yet, as shown by recent reviews (Skandalakis, Gray \& Shepherd 1960; Herlinger, 1966) they are more common than once thought, and are estimated to account for $0.5 \%$ of all gastric neoplasms. Their importance lies in their eminent curability, despite their unpromising clinical appearance.

\section{Case 1}

A 70-year-old man presented with a 3-week history of a gradually increasing swelling in the upper abdomen. His general health was good despite gross kyphoscoliosis since childhood. He had been examined by his general practitioner 1 year earlier when no mass was noted.

Examination revealed a fit man with marked kyphoscoliosis. A large mass was visible in the left upper abdomen extending down to the umbilicus and across the midline. It had a smooth surface, was of a firm consistency except for a fluctuant area in the centre of its anterior surface. There was a definite notch on its lower medial border. The mass moved with respiration. The liver was palpable but not enlarged.

Investigations. $\mathrm{Hb}, 11.9 \mathrm{~g} / 100 \mathrm{ml}$; $\mathrm{MCHC}, 32 \%$; WCC, $6900 / \mathrm{mm}^{3}$, normal differential; blood film: slight hypochromia and anisocytosis; platelet count, $229,000 / \mathrm{mm}^{3}$; coagulation screening was normal. Blood urea, electrolyte, liver function, plasma protein and electrophoretic strip, normal.

The chest radiograph showed a dorsal scoliosis. A plain radiograph of the abdomen showed a mass in the left upper quadrant of the abdomen. An intravenous pyelogram and barium enema were normal. A barium meal showed medial displacement of the stomach by a mass, with no disturbance of the rugal pattern. Isotope scanning showed that the liver was distorted by external pressure, but the spleen wasn normal. A selenomethionine abdominal scan was inconclusive. Respiratory function tests were performed and showed severe disturbance of ventilation perfusion relationships in the lung. The carbon monoxide transfer factor was $7.2 \mathrm{~mm}$, with a predicted normal of $23 \mathrm{~mm}$, and the $\mathrm{Po}_{2}$ was $62 \mathrm{mmHg}$. A diagnosis of splenic cyst was made prior to laparotomy.

At operation a tumour $20 \mathrm{~cm}$ in diameter was found, occupying the left upper quadrant of the abdomen. The mass was adherent to the left lobe of the liver, the greater omentum, the transverse colon and mesocolon, the posterior abdominal wall and the stomach. The mass was grey in colour, lobulated and cystic. One of the larger cysts was opened and 1 litre of blood-stained fluid drained. A frozen section of the cyst wall showed that it was a connective tissue tumour. The tumour was mobilized and its attachment to the greater curvature of the stomach eventually demonstrated. A cuff of the greater curvature was removed with the tumour. No mucosal defect of the cuff of the stomach was found.

Histology showed a large gastric leiomyoma undergoing cystic degeneration.

Postoperative recovery was uneventful and the patient was discharged on the sixteenth postoperative day. Respiratory function tests were repeated 1 month later and showed significant improvement. The carbon monoxide transfer factor had increased to $16.6 \mathrm{~mm}$ and the $\mathrm{Po}_{2}$ to $76 \mathrm{mmHg}$. 


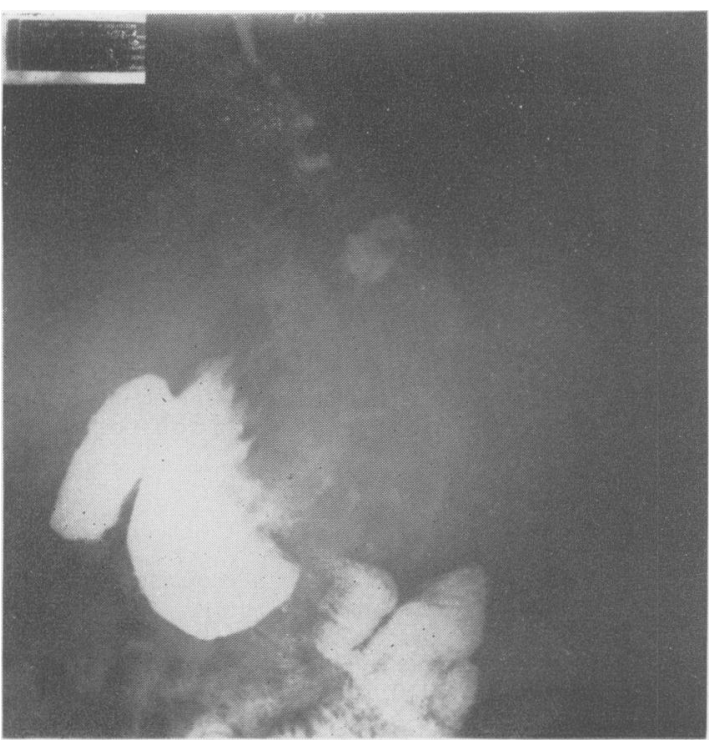

FIG. 1. Barium meal radiograph of Case 1.

\section{Case 2}

An 85-year-old lady presented with an 8-year history of intermittent episodes of right sided abdominal pain, with a more severe attack of pain 4 weeks previously. At the time of this severe episode of pain she noted a mass in her left upper abdomen. There were no previous illnesses.

On examination she was a fit lady for her age with no abnormality other than an irregular surfaced firm mass situated in the left upper abdomen with a well defined edge, extending from the midline to the loin. It was ballotable in the loin and moved with respiration. A normal liver edge was palpable below the right costal margin.

Investigations. $\mathrm{Hb}, 12 \cdot 2 \mathrm{~g} / 100 \mathrm{ml}$; WCC, $5300 /$ $\mathrm{mm}^{3}$, normal differential. Blood urea, electrolyte, liver function, plasma protein and electrophoretic strip were normal. A mid-stream specimen of urine showed no cytological or bacteriological abnormality. Chest radiography was normal. A barium enema showed left-sided diverticular disease and downward displacement of the splenic flexure by a mass. An intravenous pyelogram showed medial displacement of the left kidney and deformity of its upper pole calyces. The right kidney and remaining renal tract were normal. A provisional diagnosis of a left renal neoplasm was made.

At operation with the patient in the left lateral position, a left loin incision was made above the twelfth rib. A normal left kidney was found, but it was displaced by a large intraperitoneal mass, some $30 \mathrm{~cm}$ by $20 \mathrm{~cm}$ originating from the greater curva- ture of the stomach, but attached to the spleen, the posterior abdominal wall and the diaphragm. A clinical diagnosis of a leiomyoma was made, and the incision converted into a thoraco-abdominal one through the bed of the ninth rib. After aspirating $330 \mathrm{ml}$ of blood-stained fluid from the cystic part of the tumour, the mass was removed with a segment of diaphragm and a cuff of the greater curvature of the stomach.

Histology showed a cystic gastric smooth muscle tumour and although parts of the tumour showed mitotic activity, there was no evidence of invasion, and it was therefore considered to be a benign gastric leiomyoma.

Postoperative recovery was complicated by a wound infection which delayed her discharge until the thirty-seventh postoperative day.

\section{Discussion}

Exogastric smooth muscle tumours have been well reviewed by Skandalakis and their radiological presentations by Herlinger, yet the clinical presentation and the treatment of these tumours still presents a problem. The cases presented here represent the two most common clinical presentations, namely a symptomless mass in the upper abdomen and pain associated with an abdominal mass.

Despite extensive and detailed investigations, the first case was not diagnosed pre-operatively and serves as a reminder that although barium meal studies are the single most significant investigation in the diagnosis of this condition, absence of typical radiological signs, such as the 'cone d'attraction, does not exclude the diagnosis of exogastric leiomyomata. The second case illustrates a point made by Herlinger that the tumour can simulate a renal neoplasm through its ability to distort the calyceal pattern of the kidney as seen on intravenous pyeolgraphy. A further radiological investigation of use in diagnosis is selective angiography. This technique delineates the tumour pre-operatively, and identifies its blood supply; control of the blood supply to these frequently highly vascular tumours is one of the most important features of safe resection.

At operation or even in their pre-operative assessment, the size and multiple attachment of this type of tumour and their apparent malignancy may deter the surgeon from attempting excision. The decision to resect these two tumours was made on the basis of their mobility and absence of invasion of vital structures. Even if sarcomatous change is suspected, extensive local resection and lymph node clearance has not been found to improve the prognosis. The resection of a small cuff of stomach with the tumour as in the two cases described, is adequate.

The technique of removal of these tumours is important, for angiography has shown that though 
their blood supply is extensive it arises from vessels normally supplying the stomach, and bleeding is reduced by carrying the dissection towards the midline origin of these vessels. A thoraco-abdominal incision as used in the second case, is well suited to this lateral to medial dissection.

The ability to recognize these tumours from their gross appearance is important, for the pathologist may not be able to make a diagnosis from frozen sections of the tumour. Even in prepared histological material differentiation between benign and malignant smooth muscle tumours of this type is difficult. The clinical progress of such patients is the best indicator of the pathological nature of the original tumour, if a histological diagnosis of malignancy was not made on the operation specimen. Both the patients described here are alive and well 1 year after resection with no evidence of recurrence.

It is of interest that the first patient noted an increase in his exercise tolerance following the removal of his tumour. This was confirmed by significant improvement in respiratory function tests performed postoperatively.

That excision of these large exogastric tumours was possible and successfully performed in two patients, whom by age and respiratory insufficiency respectively carried a greater than normal operative risk supports and underlines the plea for total excision of these tumours.

\section{Acknowledgments}

We thank Mr C. J. B. Murray and Mr W. W. Slack under whose care these patients were admitted, for permission to publish these case reports and for their help with the preparation of this paper.

\section{References}

Herlinger, H. (1966) The recognition of exogastric tumours. British Journal of Radiology, 39, 25.

Skandalakis, J.E., Gray, S.W. \& Shepherd, D. (1960) Smooth muscle tumors of the stomach. International Abstracts of Surgery, 110, 209.

\section{Survival of candida septicaemia treated with amphotericin B}

\author{
A. B. S. Mitchell \\ M.B., M.R.C.P. \\ G. HosKING \\ M.B., L.R.C.P.
}

\author{
I. T. Magrath \\ M.B., M.R.C.P.
}

C. P. FARThing

M.B., M.R.C.Path.

West London Hospital, Charing Cross Group of Hospitals, London, W.6

\begin{abstract}
Summary
Candida albicans septicaemia occurred in an alcoholic woman who had severe hepatic dysfunction, and was further debilitated by multiple cardiac arrests, prolonged artificial ventilation, bronchopneumonia and $P$. aeruginosa septicaemia. She received broad spectrum antibiotics and corticosteroids, and needed prolonged intravenous cannulation. In vitro, a specific defect was demonstrated in her cell-mediated immune response to candida antigens. Examination of her serum showed normal immunoglobulins, and an adequate agglutination titre to these antigens.

Amphotericin B (800 mg intravenously over 19 days) was administered, and she survived. Hypokalaemia, anaemia, and peripheral neuropathy complicated
\end{abstract}

therapy, and thrombophlebitis of a cannulated peripheral vein necessitated a vena caval catheter. There was no significant deterioration of renal function.

Toxic effects of other drugs were encountereddeafness following kanamycin, an encephalopathy when blood levels of carbenicillin were boosted by probenecid, and cardiac arrest when diazepam and phenobarbitone were administered to control an epileptic fit and delirium tremens. Prolonged intravenous cannulation was complicated by staphylococcal septicaemia.

A year later she died of peritonitis. Chronic pancreatitis was complicated by ascites, which became infected by $E$. coli. No residual $C$. albicans infection was found at necropsy. 